\title{
Sociocultural Aspects of Health Promotion in Palliative Care in Uganda
}

\author{
James Mugisha
}

\section{Abstract}

Despite its vital importance, health promotion has not occupied its due place in public health in Uganda. The country is engulfed into a rising wave of both communicable and noncommunicable conditions. This rising burden of both communicable and non-communicable conditions turns health promotion and palliative care essential health care packages; though there is little to show that these two important programs are getting vital support at policy and service delivery levels. A new theoretical framework that is anchored into sociocultural issues is essential in guiding the design and delivery of both health promotion and palliative care in Uganda. The salutogenic theory puts socio-cultural issues at the centre of developing health promotion and palliative care and, seems to solve this dilemma. In this chapter, illustrations from indigenous communities in Uganda are employed to demonstrate the challenges to the health promotion and palliative care agenda in the country and how they can be addressed. Uganda Ministry of Health should develop robust structures within public health for development of health promotion and palliative care in the country.

J. Mugisha $(\bowtie)$

Faculty Arts and Social Sciences, Department of Sociology and Social Administration, Kyambogo University, Kampala, Uganda
Research should be conducted on the effectiveness of the current strategies on health promotion and palliative care and their cultural sensitivity and appropriateness. Given the limited resources available for development of health care in Uganda, as an overall strategy, health promotion and palliative care should be anchored in public health and its (public health) resources.

\section{Keywords}

Social aspects $\cdot$ Salutogenesis $\cdot$ Health promotion $\cdot$ Palliative care $\cdot$ Uganda

\subsection{Background}

There is a dramatic shift in low-income countries from communicable to non-communicable diseases [1-3]. As noted, by 2030, the burden of non-communicable diseases, including neuropsychiatric disorders, will constitute seven of the ten leading causes of disease burden globally and its impact will be more felt in low-income countries where health systems are more fragile as compared to the ones in high-income countries $[1,4,5]$. It is estimated that within a generation, the share of disease burden attributed to non-communicable diseases in some poor countries of the world will exceed $80 \%$, rivalling that of rich countries 
[3]. And, this burden is likely to affect more the younger generation in poorer resource contexts than in the high-income countries [3].

The most prevalent globally of these noncommunicable conditions are: cardiovascular diseases, cancers, chronic respiratory disease and diabetes [6]. And, a similar trend seems to be unravelling in Africa [6]. Globally, it is estimated that these conditions, contribute to large mortality, accounting for 36 million deaths in 2008 (63\% of total fatalities) [7]. Majority (four-fifths) of these deaths occur in LMICs [7]. It is also estimated that, if these trends go unchecked (as it is the case in most LMCs), by 2030, deaths due to NCDs will be the most common causes of mortality in low-income countries. More specifically, diabetes cases in sub-Saharan Africa are projected to increase from $4.8 \%$ prevalence $(19.8$ million) in 2013, to $5.3 \%$ (41.5 million) in 2035 (IDF, 207). In the same vein, cancer cases are also projected to nearly double $(1.28 \mathrm{~m}$ new cases and 970,000 deaths) by 2030 (in 2012 there were 645,000 new cases and 456,000 cancer-related deaths in Africa) [8]. The most obvious risk factors are a combination of increasing and ageing populations, the adoption of risk-factor lifestyles (largely due to sedative life style), and deficient diagnostic, preventative and curative treatment services [9].

Unfortunately, in many of the low-income countries that have started to witness this shift, the health system is not prepared enough to deal with this challenge [2, 3]. Many of the noncommunicable conditions are chronic in nature necessitating the need for a fundamental reprograming of the public health sector and palliative care.

\subsection{The Public Health and Palliative Care Context in Africa}

The need for palliative care in low-income countries in general and Africa in particular is already overwhelming [10, 11]. Evidence is available that there is a large gap between the number of people in need of palliative care services and those who are able to actually receive it [12]. At present, only a few countries have any form of palliative care program and this gap may be larger in Africa [7]. There are no obvious strategies to meeting this need due to already overstretched health systems in low-income countries and the ever-increasing political dilemmas (such as political turmoil and mismanagement) that affect service delivery.

Suggestions have been made that public heath can effectively work in tandem with palliative care [13]. It is possible for public health to enter deeply into palliative care narratives and establish strong relationships to improve the current service delivery mechanisms of both programs (public health and palliative care) [13]. The essence here is using a public health approach in palliative care. This presupposes fundamentally building community capacity to own and work on its health issues. Such approaches have large benefits of being cost effective, empowering; improve coverage of services to the general population and are sustainable [13]. All that is needed is conceptual clarity [13]. The revised WHO definition of palliative care is anchored into public health and seems to take care of these concerns [13]. Palliative care is defined as:

Palliative care is an approach that improves the
quality of life of patients and their families facing
the problems associated with life-threatening ill-
ness, through the prevention and relief of suffering
by means of early identification and impeccable
assessment and treatment of pain and other prob-
lems, physical, psychosocial, and spiritual
(Whitelaw and Clerk [13], p. 4).

The notion of prevention and early detection of suffering is highlighted in this definition and is key to the public health agenda. This understanding did not get the due emphasis in the previous public health efforts [13]. Current public health efforts should have a new outlook that focuses on a comprehensive person with psychical, psychosocial and spiritual needs.

Taking as comprehensive approach to health service delivery demands for a lot of resources. In many low-income settings however, public health has relatively compelling volume of resource as compared to other sectors and these resources 
could be used in shaping the future direction of the palliative care policy $[2,13]$. Deep reflections are needed here to create a secondary deployment (where palliative care is "infused" into public health) to create better synergies [13]. Moreover, within this new thinking, the need for palliative care should be understood as a public health issue [13]. This evidence (of infusing palliative care into public health care) is already available in some countries; though still in a few of them [13].

\subsection{Health Promotion and Salutogenesis: Concepts and Theory}

The concept health promotion has had more extensive use in the developed world as compared to the low-income countries. Health promotion in Africa in general and Uganda in particular is not well articulated in the public health agenda. In this chapter we shall adopt the World Health Organisation (WHO) definition of health promotion: "Health promotion is the process of enabling people to increase control over, and to improve, their health" [14]. The biggest challenge for health promotion in Africa and Uganda in particular has been: (a) lack of frameworks and models for classifying activities and determine the scope of health promotion [15] and, (b) most people in Africa have poor health status and therefore it is difficult to sustain development and economic viability of most health programs [2]. Due to the above-mentioned challenges, to a larger extent, most countries in Africa have had their focus on curative care, they have minimal resources to satisfy this sector (curative sector) and because of this reason, health promotion has been forgotten.

\subsection{Salutogenesis in Health Promotion and Palliative Care in Uganda:Theoretical and Status Issues}

Within the field of health promotion, perspectives have emerged. Some of these include positive psychology and salutogenesis. The interest in this chapter will be salutogenesis though positive psychology, which is also of much relevance here. The originator of salutogenesis (Aaron Antonovsky) described health systems in the Western world as "pathogenic" [16]. His description seems to be of much relevancy to the health systems in the developing world such as Uganda. Simply put, Antonovsky [17] referred to this malaise in the health system as "disease care system" ([17], p. 12). His perspective is a sharp opposition to the pathogenic orientation, which is dominant in Western medical thinking [18]. "Antonovsky rejected a dichotomous categorisation of the health status (e.g., well vs. diseased, healthy vs. ill as inappropriate) to represent the complexity of health status" [18]. His view was that health is more reasonably understood as a continuum; every person is at a given point in time somewhere between health and disease poles in the continuum [18]. In this regard, he coined the "construct of generalised resources against stress" (which is defined as a property of the person, a collective or situation which, as evidence or logic has indicated, facilitated successful coping with inherent stressors of human existence" ([17], p. 15; [18], p. 326). Related to this the construct of "sense of coherence", which is a generalised orientation towards the world, which perceives it, on ease/health continuum, as comprehensible, manageable and meaningful ([17], p. 15). "When confronted with a stressor, people with a strong sense of coherence are likely to be motivated to cope (meaningfulness), to believe that coping resources are accessible" (manageability) [18]; also see Antonovsky [17]. This thinking is useful for those at the dyeing stage of their life.

Since hospitals are traditionally characterised by an orientation to diagnosing, curing and caring for severely ill people [19] they have a limited contribution to the public health agenda. This understanding calls for new frameworks for health promotion and palliative care in Uganda. The curative approach fails to recognise that death, dying, loss and care giving exist to some extent beyond the domains of individualistic therapeutic intervention and a public health care approach brings better results [13]. It requires the 
reorientation of health services in Uganda into more public health and health promotion domains; as demanded by the Ottawa Charter [14]. This has not happened to a remarkable degree yet in many countries.

\subsection{The Cultural Context and Issues Related to Death and Dying in Uganda}

\subsubsection{Meaning of Death}

During the dyeing process, many people within indigenous communities are preoccupied with the meaning of Good Death. Drawing from sociality, good death culturally means dying while surrounded by people, especially, your children, wives(s) and close relatives. Though having less pain is part of good death this is not the most important consideration of good death as emphasised by modern health workers. In our study in Mpigi [20], we saw our informants having a negative attitude against long periods of hospitalisation that are normally synonymous with chronic diseases (also see $[20,21])$. Long stay in hospital is seen as affecting sociality since the patient is normally away from his/her children, spouse and close family members. What was evident from the narratives expressed by people in our project in Mpigi is that the cultural conception of death was different from the "scientific view" of good death and this has implications on the way health promotion and palliative care should be delivered. Searching for meaning in life is part of the salutogenic approach and this makes it important for any health service to focus on the subjective experience of the people targeted. Within salutogenesis, it is postulated that there is a connection between spirituality (religion and meaning making) and health. Within salutogenic and the health promotional framework, dimensions of health are referred to in terms of the physical, mental, social and spiritual; a notion of huge relevance to health and palliative care programs in Uganda $[22,23]$. This calls for comprehensive and integrated programs that can address physical, mental, social, and spiritual aspects of human functioning and, these are inextricably intertwined.
Though Uganda is getting more urbanised (over $76 \%$ of the Ugandans live in rural area), the influence of culture (and tradition) is still so strong among indigenous communities in Uganda. Health promoters have to manage diversity; the patient, family and the community where they live [24]. The cultural values of the patient and family within the locations/contexts must be given attention [24]. Uganda is quite cosmopolitan in terms of tribes (over 50 tribal groupings), age, gender and other socioeconomic variables. In many of the societies, health promotion and palliative care should pay attention to these multiple components including race, ethnicity, gender, age, differing abilities, sexual orientation, religion, spirituality, and socioeconomic status [24]. The beliefs, norms and practices should be the target of the health promotion activities as they are likely to guide behavioural responses, decision-making and action and other key variables related to acceptability of health promotion and palliative care.

\subsection{Aetiology and Cultural Frameworks}

Within the salutogenic theory, existential issues are given due attention. One goes into meaning making, contact inner feels in order to mobilise generalised resources. The subjective experience of diseases such as cancer and HIV/AIDS is cultural based [25]. They are cognitively and linguistically expressed within cultural frameworks and therefore the medical model (pathogenesis) becomes less meaningful to those afflicted by disease. Among indigenous communities, causality of disease is largely attributed to external factors or different aspects in the natural environment, both living and/or non-living [25]. Studies undertaken in the field of mental health indicate that over $80 \%$ of the people in Uganda seek help from spiritual/traditional healers [20, 26, 27]. Because of the differences in perspectives between modern and traditional healers, there develops polarised relationships between the two (modern and traditional healers). This polarisation normally delays appropriate treatment seeking: 
Cancer is about a neighbour who might not be happy with your achievements. The medical stories don't make sense to our people and that is why they keep home till death (Personal Communication, Anthropologist Makerere University Medical School).

There is always a failure by the health system to undertake a meaningful co-construction between the patient, the family, the traditional healers and the modern health systems. The palliative care and health promotion field fail to deal with medical domination of the traditional system. Many of the people they are targeting within indigenous communities have existential needs for which they have little trust in the modern health system. With the salutogenic field, it is postulated that the way people view themselves and the world has implications for their health and more importantly, their quality of life. Hence, the need to shift attention to the subjective experience of the people and to change their existing perspectives to health care. We cannot afford to ignore the backyard issues that seat at deepest part of people's values and belief systems. Our study in northern Uganda; the Wayo-Nero Strategy (https://www.mhinnovation.net/innovations/wayo-nero-strategy) aimed at reducing the treatment gap for mental disorders by utilising indigenous institutions in post-conflict areas. Many people targeted in the three districts in northern Uganda were able to access services using this strategy. The Wayo (aunti)-Nero (uncle) are traditional counsellors and their services were harnessed to deliver modern health care but also spiritual care. This made the interventions more acceptable to the people since we were using a cultural resource.

\subsubsection{Communication About Death}

The subjective experience of diseases such as cancer and HIV/AIDS is culturally based [25]. They are cognitively and linguistically expressed within cultural frameworks. Again in our research work in Mpigi, we see a challenge where indigenous communities have great fear and respect for death and never refer to death directly. Instead, they circumvent around its (death) meaning using their oral skills. In our study in Mpigi among indigenous communities in Uganda (see [19, 20]), our research topic was on the meaning of completed suicide. We as researchers were not born in this culture and never knew that "Baganda" do not refer to death directly. Once we did that cultural tension emerged. Death as a subject cannot easily be avoided in palliative care. However, discussing it among indigenous communities must be done with a lot of cultural sensibility [20]. In our project, we adapted the concepts that could mean death but less offence to culture and tradition (see $[19,20])$. This experience is of high relevance to the current health promotion and palliative care workers in Uganda.

\subsubsection{Masculinity and Help Seeking}

Society always sets different expectations for men and women [20]. The cultural meaning of a "real man" sets expectations that men even under great pain should undertake controlled emotional expression (should suppress pain inwards) (see $[19,20])$. But this may again come with other risks as the pain may become more severe; patients may become depressed or traumatised by diseases among other risks [20]. Culturally, accepting uncontrolled emotional expression is turning into a "woman". The most obvious challenge with masculinity in this context is that it limits sharing of information between the client and the palliative health care worker because of the high possibly of normalising pain; one has to be a man. The man is expected to be tough and this has to be demonstrated even in situations of great pain. Interestingly, strict adherence to masculine norms in indigenous communalities is rewarded by society with respect before and after death. "The man is praised for being a man and not a woman and that means keeping quite over most of the pain" (Personal Communication with Senior Lecturer Anthropology Makerere University). Too much emotional outpour is culturally disrespected and only expected of women. "For a woman it is ok to cry before the public 
but for the man you are expected to do otherwise" (Senior Lecturer Anthropology Makerere University).

Masculinity also comes with a belief in selfreliance, the need to do things by yourself and these trends can be seen in urban areas where communalism has lost grip on society and there is more individualism. In our study in Mpigi [20], we established individualistic traits in urban areas where people are more inclined to self-efficacy. Researches in mental health indicated that, those inclined to masculine ideology and, have strong individualistic construes rarely seek formal health care. Palliative care to them has a connotation of communalism-sharing your problems with others. "Yah, there are those who want to die with their private life and the way our palliative care is structured with several nurses and village health workers, it cannot work for such people" (Lecturer Social Work Kyambogo University).

\subsubsection{Community Gate-Keeping and Health Promotion}

Most studies largely undertaken in the developed world have looked at the notion of gatekeeping in terms of research-the structures, which the researcher(s) has to deal with to access potential respondents for the study [20]. In this study I take a different view. I look at gate-keeping agencies as community structures, which the program has to deal with to access clients [20]. In indigenous communities, cultural institutions/structures are the custodians of culture and play a major role in its survival [20]. They also play a major role in the survival of community members including infirmities that might befall individuals, families or the larger community and they are always called upon when negative life events befall a community [28]. Many of the cultural leaders are at the same time the political leaders of the community as they play multiple roles [29, 30]. Several studies conducted in Uganda indicated that they increase acceptability and coverage of community programs $[29,30]$. They have been instrumental in popularising HIV/AIDS [31] and malaria campaigns at community level. Though palliative care has exploited some of the community leaders attached to the HIV/AIDS programs, this has been undertaken to a limited extent and many of them have not been trained in palliative care as a methodology. In many communities in Uganda, there are no community-based palliative care workers despite the program having a national coverage. "The coverage of community workers is still marginal and needs to be addressed. And many of them lack the core palliative care skills and health promotion. HIV/AIDS is not necessarily palliative care" (Personal Communication, Lecturer Palliative Care Kyambogo University, Uganda).

In our Wayo-Nero Mental Health Care Project, we managed to bridge the treatment gap for common mental disorders using the Wayo (aunt)Nero (uncle) as traditional institutions (https:// www.mhinnovation.net/innovations/wayo-nerostrategy). The Wayos and Neros used their influence as cultural leaders to deliver the program including health messages. There are lessons to learn from the Wayo-Nero project by the health promoters in palliative care in Uganda in increasing addressing sociocultural issues in health.

\subsection{Looking at the Future}

The salutogenic field provides an important theoretical framework for health promotion in Uganda. "Africans are notoriously religious, and each people has its own religious system with a set of beliefs and practices" [32]. Mbiti further observed that "religion permeates into all the departments of life so fully that it is not easy or possible always to isolate it" [32]. And most of the time African people are entangled in both modern and traditional religious systems [20, 32]. Quite more modern religious writers such as Gyekye [33] observed that African heritage is intensely religious. "The African lives in a religious universe: all actions and thoughts have a religious meaning and aspired or influenced by a religious point of view ([33], p. 3). The views expressed above by the two African writes Mbiti [32] and Gyekye [33] though have been in print 
for a while still largely represent the religious systems of African people. They indicate the religious standpoint of African people and any effective program should address their spatial needs [20]. There is a possibility to use the current cultural resources (both modern and traditional) to improve public health and the delivery of health promotion and palliative care. HIV/AIDS, malaria and other communicable diseases have attracted quite a lot of resources from the donor community. Some of these resources can be used to develop a national framework for delivery of health promotion and palliative care in the country. Indigenous (e.g. spiritual healers and herbalists) and modern cultural (e.g. churches and church leaders) resources could be tapped into to deliver both health promotion and palliative care more effectively and with a high level of cultural acceptability. Drawing again from our salutogenesis framework, these historical roots that are embedded on religion and religious associations have implications in strengthening the members of these societies in their sense of coherence [34]. Cultures seem to define the resources that are appropriate to deal with a stressful situation [34]. The cultural context is likely to shape the type of the stressor experienced by the individual and also the choice of appraisal and coping strategy employed [34]. The spiritual/religious social networks can be important sense of community coherence.

The Ottawa conference in 1986 called for reorientation of health services. It was further observed that there has been slow progress in making health promotion a core business for health services and there was a need to reframe, reposition and renew efforts in this field [35]. Part of these efforts includes being more active in health systems development $[35,36]$. This slow development is more felt in Africa and Uganda in particular. The Uganda Ministry of Health needs to make pragmatic steps in making health promotion a priority sector within the ministry. This is more critical today with the aging of the population in Uganda and the rising impact of chronic diseases in the country [35]. New policies and budget frameworks that are sufficient and efficient should be developed and operationalised.
Unfortunately, many Ministries of Health in Africa and this is also the case with Uganda lack a health promotion structure [15]. As noted these Ministries always have an IEC Unit, perform traditional health education functions and not tied to the overall global framework of health promotion [15]. Health promotion should not be seen as an added cost but as a cost saving strategy (especially when one focuses on disease prevention, patient empowerment and community management and participation).

Research should be undertaken to establish the cost and effectiveness of health promotion activities that are part of the health promotion and palliative care agenda currently and in future [35]. Government agencies such as universities and especially schools of Public Health should take leadership in this. The World Health Organisation's Ottawa Charter for Health Promotion from 1986 is still the gold standard for health promotion worldwide [36]. Academic institutions (though none exists in Uganda) can set up centres of excellence on health promotion to foster critical thinking about health promotion in the country. They can also ensure that the gold standard envisaged in the Ottawa charter is engendered though this has to be done with caution [15]. The Alma Ata Declaration is deemed more impactful on the continent due to its strong focus on primary health care [15] while the Ottawa conference is quite lacking in this since its focus was more on industrialised countries. It has argued that that there is a possiblity for the two instruments (Alma Ata Declaration and Ottawa Charter) to cross-fertilise each other. The Alma Ata Declaration can be used for comprehensive primary health care while the Ottawa Charter can be more useful in tacking the double burden of emerging non-communicable and communicable diseases [15]. This also requires changing the political will and ideology in many countries [36].

While local government are rich in local context which can improve the cultural sensitivity of their programs, they at the same time lack the required resources especially funds to deliver public health interventions [15]. However, a public-private partnership can boost the amount 
of resources available at the local government level (Alma Ata Declaration).

Overall, efforts should be made to ensure that health promotion is embedded into all aspects of life, including home, work leisure and within health care [36]. A special focus on the social aspects of health promotion and palliative care makes this agenda feasible. Watson [37] suggested that those involved in health promotion should ensure: (a) creation of a healthy working environment (c) integrating health promotion into daily activities and (c) reaching out into the community. The crosscutting theme in all these three aspects is culture and social aspects of health promotion, becomes an important theme. Given the limited resources in Uganda, it might be difficult to work with all units in the health sector at the same time. However, we could go piecemeal until all vital units in the heath sector are covered. Even in the developed countries, these variations exist despite having a relatively larger volume of resources. Hospitals still take a lion's share of the Uganda health sector budget and primary health care where much of the health promotion takes place is still neglected. This should be reversed. The words of John Catford are also very informative here, "We look in eager anticipation to see how Africa moves ahead in closing the implementation gap in health promotion...Although Africa may light the way, the rest of the world will also need to shoulder the task" ([35], p. 3). These words have been interpreted to imply that the international community should play a major role in helping Africa to close the gap in health promotion [15]. While understanding is important, the Western world should not transpose health promotion packages from their countries as it normally happens with many of the development assistance programs in the health sector [15]. As noted, high-income countries differ a lot from low-income countries in key aspects especially in their individualist/communitarian orientations [15]. A home grown package that takes into account the social aspects in health promotion and palliative care should be developed and propagated. As seen in our findings, communalism is still the mainstay in social organisation of our study communities. The health promotion agenda should be based on communalism rather than an individualist Eurocentric health promotion discourse and practice [15]. This thinking does not serve to connote that there should be only an African public or health promotion agenda. However, it is an attempt to look at public health and health promotion in Africa with contextual eyes, cultural sensitivity and lastly in its own right.

\subsection{Conclusion}

Uganda's quest for palliative care national wide is based on false premises and constitutes a categorical fallacy. The current model spearheaded by hospitals to deliver health promotion and palliative care misses the critical components that are vital in this field. Individuals and families are not empowered to take charge of their health needs and resources to support care. The salutogenic theory takes care of these dilemmas and takes health promotion and palliative care into communities. The existing resources for public health in Uganda should be synergistically tapped into to develop health promotion and palliative care through a community based model.

\section{Take Home Messages}

- The need for palliative care in Uganda and other low-income countries is real and growing.

- The existing medical model(s) that have historically informed the development and delivery of health service in Uganda are faced with insurmountable challenges and therefore there is need for a paradigm shift.

- Culture stands in the road to providing effective palliative care and this challenge should be addressed through designing programs that are culturally sensitive and acceptable to individuals and communities.

- Uganda Ministry of Health needs to move away from tokenistic approaches to health promotion and palliative care to comprehensive programs. Health promotion and palliative care should be accorded their due right-not to remain small units in the health education 
sector at the Uganda Ministry of health but become fully-fledged departments.

- Education institutions in the country should build capacity for research in this field and also train specialists in health promotion and palliative care.

\section{References}

1. Marais DL, Petersen I. Health system governance to support integrated mental health care in South Africa: challenges and opportunities. Int J Ment Health Syst. 2015;9:14. https://doi.org/10.1186/ s13033-015-0004-z.

2. Mugisha J, Ssebunnya J, Kigozi FN. Towards understanding governance issues in integration of mental health into primary health care in Uganda. Int J Ment Health Syst. 2016;10(1):25.

3. Bollyky TJ, Templin T, Cohen M, Dieleman JL. Lower-income countries that face the most rapid shift in noncommunicable disease burden are also the least prepared. Health Aff (Millwood). 2017;36(11):1866-75. https://doi.org/10.1377/ hlthaff.2017.0708.

4. Mugisha J, Abdulmalik J, Hanlon C, Petersen I, Lund C, Upadhaya N, Ahuja S, Shidhaye R, Mntambo N, Alem A, Gureje O, Kigozi F. Health systems context(s) for integrating mental health into primary health care in six Emerald countries: a situation analysis. Int J Ment Health Syst. 2017;11:7. https://doi. org/10.1186/s13033-016-0114-2.

5. World Health Organization. The global burden of disease: 2004 update. Geneva: WHO; 2004.

6. WHO. Mental health action plan 2013-2020, Geneva; 2013.

7. Alwan A, et al. Monitoring and surveillance of chronic noncommunicable diseases: progress and capacity in high-burden countries. Lancet. 2010;376:1861-8.

8. International Agency for Cancer Research. Latest world cancer statistics - GLOBOCAN 2012: estimated cancer incidence, mortality and prevalence worldwide in 2012, Geneva; 2012.

9. Peters R, Ee N, Peters J, Beckett N, BoothA, Rockwood $\mathrm{K}$, Anstey KJ. Common risk factors for major noncommunicable disease, a systematic overview of reviews and commentary: the implied potential for targeted risk reduction. Ther Adv Chronic Dis. 2019;10:2019. https://doi.org/10.1177/2040622319880392.

10. World Health Organisation. Global status report on non-communicable diseases 2014. Geneva: World Health Organisation; 2014.

11. Currow DC, Allingham S, Bird S, Yates P, Lewis J, Dawber J, Eager K. Referral patterns and proximity to palliative care inpatient services by level of socioeconomic disadvantage. A national study using spatial analysis. BMC Health Serv Res. 2012;12:42.
12. Hawley P. Barriers to access to palliative care. Palliat Care Res Treat. 2017;10:1178224216688887. https:// doi.org/10.1177/1178224216688887.

13. Whitelaw S, Clerk D. Palliative care and public health: an asymmetrical relationship? Palliat Care Res Treat. 2019;12:1178224218819745. https://doi. org/10.1177/1178224218819745.

14. WHO. Ottawa charter for health promotion. In: First international conference on health promotion, Ottawa 21 November, 1986. WHO/HPR/HEP/95.1. 1986. [Last accessed on 2014 May 10]. http://www.who.int/ healthpromotion/conferences/previous/ottawa/en/in dex4.html.

15. Dixey R. After Nairobi: can the international community help to develop health promotion in Africa? Health Promot Int. 2014;29(1):185-94. https://doi. org/10.1093/heapro/dat052.

16. Joseph S, Sagy S. Positive psychology in the context of salutogenesis. In: The handbook of salutogenesis. Cham: Springer; 2017. Chapter 10.

17. Antonovsky A. The salutogenic model as a theory to guide health promotion. Health Promot Int. 1996;11:11-8.

18. Quehenberger V, Krajic K. Applications of salutogenesis to aged and highly-aged persons: residential care and community settings. In: The handbook of salutogenesis. Cham: Springer; 2017. Chapter 31.

19. Mugisha J. Positioning for safety. Attitudes and cultural responses towards suicide among the Baganda, Uganda. PhD Thesis. Norwegian University of Science and Technology (NTNU), Trondheim, Norway; 2012.

20. Kikule E. A good death in Uganda: survey of needs for palliative care for terminally ill people in urban areas. Br Med J. 2003;327(7408):192-4. https://doi. org/10.1136/bmj.327.7408.192.

21. Eriksson M, Lindström B. Antonovsky's sense of coherence scale and the relation with health: a systematic review. J Epidemiol Community Health. 2006;60(5):376-81.

22. Eriksson M, Lindström B. Bringing it all together: the salutogenic response to some of the most pertinent public health dilemmas. In: Morgan A, Ziglio E, Davies M, editors. Health assets in a global context: theory, methods, action. New York: Springer; 2010. p. 339-51.

23. Kumar S, Preetha GS. Health promotion: an effective tool for global health. Indian J Community Med. 2012;37(1):5-12. https://doi.org/10.4103/0970-0218. 94009.

24. Haider S, Ahmad J, Ahmed M. Identifying barriers to implementation of health promoting schools in Pakistan: the use of qualitative content analysis and fuzzy analytic hierarchy process. Int J Adv Appl Sci. 2018;5(4):56.

25. Okello ES. Cultural explanatory models of depression in Uganda. Doctoral dissertation, Karolinska University and Makerere University. 2006. http:// mak.academia.edu/ElialiliaSOkello/Papers/634784/ 
Cultural_explanatory_models_of_depression_in Uganda.

26. Ovuga O. Depression and suicidal behavior in Uganda: validating the response inventory for stressful life events (RISLE). Doctoral thesis, Karolinska Institutet; 2005.

27. Stockholm, Sweden, and Makerere University, Faculty of Medicine, Kampala, Uganda. http://publications.ki.se/jspui/bitstream/10616/39769/1/thesis. pdf.

28. Menkiti I. Person and community in African traditional thought. In: Wright RA, editor. African philosophy: an introduction. New York: University Press of America; 1984.

29. Katabarwa NM, Richards FO, Ndyomugyenyi R. In rural Ugandan communities the traditional kinship/ clan system is vital to the success and sustainment of the African Programme for Onchocerciasis Control. Ann Trop Med Parasitol. 2000;94(5):485-95. https:// doi.org/10.1080/00034983.2000.11813567.

30. Katabarwa M, Habomugisha P, Eyamba E, Agunyo $\mathrm{S}$, Mentou C. Monitoring ivermectin distributors involved in integrated health care services through community-directed interventions - a comparison of Cameroon and Uganda experiences over a period of three years (2004-2006). Trop Med Int Health. 2010;15(2):216-23. https://doi. org/10.1111/j.1365-3156.2009.02442.x.

31. Muyinda H, Kengeya J, Pool R, Whitworth J. Traditional sex counselling and STI/HIV prevention among young women in rural Uganda. Cult Health Sex. 2001;3(3):353-61.

32. Mbiti JS. Introduction to African religion. Rev. ed. Botswana: Heineman Educational Publishers; 1991.

33. Gyekye K. African cultural values. An introduction. Ghana: Sankofa Publishing Co; 1996.

34. Braun-Lewensohn O, Sagy S. Salutogenesis and culture: personal and community sense of coherence among adolescents belonging to three different cultural groups. Int Rev Psychiatry. 2011;23(6):533-41.

35. Catford J. Editorial. Turn, turn, turn: time to reorient health services. Health Promot Int. 2014;29(1):1-4.

36. Ziglio E, Simpson S, Tsouros A. Health promotion and health systems: some unfinished business. Health Promot Int. 2011;26(2):216-25.

37. Watson M. InnovAiT. Education and inspiration for general practice principles of palliative care. 2008; 1:4. https://doi.org/10.1093/innovait/inn037

Open Access This chapter is licensed under the terms of the Creative Commons Attribution 4.0 International License (http://creativecommons.org/licenses/by/4.0/), which permits use, sharing, adaptation, distribution and reproduction in any medium or format, as long as you give appropriate credit to the original author(s) and the source, provide a link to the Creative Commons license and indicate if changes were made.

The images or other third party material in this chapter are included in the chapter's Creative Commons license, unless indicated otherwise in a credit line to the material. If material is not included in the chapter's Creative Commons license and your intended use is not permitted by statutory regulation or exceeds the permitted use, you will need to obtain permission directly from the copyright holder. 